\title{
Inteligencia Colectiva en el aula. Un paradigma cooperativo Collective Intelligence in the classroom. A cooperative paradigm
}

\author{
Ángel Fidalgo-Blanco ${ }^{1}$, María Luisa Sein-Echaluce ${ }^{2}$, Francisco J. García-Peñalvo ${ }^{3}$ \\ angel.fidalgo@upm.es, mlsein@unizar.es, fgarcia@usal.es
}

${ }^{1}$ Departamento Ingeniería Geológica y Minera Universidad Politécnica de Madrid Madrid, España

\author{
${ }^{2}$ Departamento de Matemática Aplicada \\ Universidad de Zaragoza \\ Zaragoza, España
}

\author{
${ }^{3}$ Departamento de Informática y Automática \\ Universidad de Salamanca \\ Salamanca, España
}

\begin{abstract}
Resumen- El principal principio de la Inteligencia colectiva se basa en que un grupo siempre puede generar más inteligencia que una sola persona de esos grupos. Este planteamiento se puede aplicar a cualquier grupo (físico o virtual) que persiga un objetivo común. Este es el caso del alumnado que está matriculado en una misma asignatura: es un grupo y comparte un objetivo común. De forma habitual el aprendizaje (destreza cognitiva de alto nivel) se plantea de forma individual, así como la inteligencia. En este trabajo se aplican modelos de Inteligenca Colectiva desarrollado en las clases de aula y soportado por una red social. De tal forma que permita cooperar a todo el alumnado de la asignatura para su objetivo común: el aprendizaje.
\end{abstract}

\section{Palabras clave: Inteligencia Colectiva; Redes Sociales; Cooperación.}

Abstract- The main principle of collective intelligence is based on one group can always generate more intelligence than a single person within those groups. This approach can be applied to any group (physical or virtual) that pursues a common goal. This is the case of students who are enrolled in the same subject: it is a group and shares a common goal. Usually learning (high level cognitive skills) is posed individually as well as intelligence. In this paper, we apply models of collective inteligence, which have been developed in classrooms and supported by a social network. In such a way that it allows to cooperate to all the students of the subject for its common objective: the learning.

Keywords: Collective Intelligence; Social Networks; Cooperation.

\section{INTRODUCCIÓN.}

Las sociedades nacen por la acción de un impulsor que afecta a los valores dominantes imperantes hasta el momento; es decir, afecta a la forma en que se realizan las actividades, a los productos, servicios y a las demandas. Todo esto afecta de forma considerable a las organizaciones, algunas desaparecen porque no saben adaptarse a esa nueva sociedad, otras consiguen transformarse y, lo más habitual, es que surgen nuevas organizaciones creadas con los valores y características propias de la nueva sociedad (Sein-Echaluce, Fidalgo-Blanco, \& García-Peñalvo, 2016).

La escuela, entendiendo esta como cualquier centro de formación, se creó y diseño en la sociedad industrial, su objetivo era formar al mayor número de personas posibles, al menor coste y con la mayor eficacia (tanto en tiempo como en nivel de conocimiento). La escuela se vertebra entorno al profesorado, que hace las veces de experto tanto en una determinada materia como en la forma de impartir la docencia. El conocimiento a transmitir es jerárquico y por tanto el alumnado se concibe como un sujeto pasivo que debe asimilar los conocimientos al ritmo, criterio y capacidad del profesorado. Básicamente, el modelo se basa en que el profesorado es seleccionador, creador de conocimiento (algunas veces) y emisor del mismo. Mientras que el alumnado es un sujeto pasivo que asimila el conocimiento.

Es evidente que la escuela ha cambiado desde su creación, nuevos conocimientos, nuevas herramientas, mayor facilidad de acceso y nuevas metodologías formativas. Sin embargo, lo que ha permanecido invariable es en la centralidad del profesorado, el profesorado elige el método, que actividades debe hacer el alumnado y los recursos a utilizar. El alumnado puede estar más o menos activo, más o menos motivado, pero todo lo que hace es para que se produzca un aprendizaje individual. Por esta razón, una gran parte del éxito o fracaso de un determinado alumno depende de su implicación, de su responsabilidad y de la realización de lo que indique el profesor en todo momento. Y a su vez, de la calidad del profesor depende la motivación, el interés y la participación que traslade a su alumnado.

Frente a esta jerarquía unidireccional de conocimiento surgen organizaciones que plantean que el conocimiento tiene múltiples flujos, focos y con un planteamiento en red (Siemens, 2005). Son varias las denominaciones que se han dado a este tipo de visión: organización en red, organización cooperativa, comunidades de práctica, comunidades virtuales de aprendizaje (Ramírez-Montoya \& García-Peñalvo, 2018). Independientemente de la denominación, una característica común es que el éxito de las mismas depende de la capacidad para actividad la inteligencia de todos sus miembros (Pör, 2008).

Sin embargo, no solamente se tiene que conseguir que los miembros de una organización cree conocimiento, debe ser capaz de gestionarse (Fidalgo-Blanco, Sein-Echaluce, \& García-Peñalvo, 2014, 2015). La gestión del conocimiento se basa en la generación, identificación, clasificación, organización y utilización del conocimiento creado en una organización, pero no solo de una persona (la más experta de la organización) sino de todas las que forman parte de la misma (Nonaka \& Takeuchi, 1995). La gestión del conocimiento supone un mejor desempeño de la organización a través del aprendizaje continuo de sus miembros. A mayor aprendizaje se produce una mayor capacitación y, por tanto, un mejor desempeño.

Teniendo en cuenta esta tendencia, en la escuela se debería considerar todo el conocimiento creado por todos sus miembros, trasladando esto a un aula; se debería gestionar el 
conocimiento de todos sus miembros (profesorado y alumnado) para que se produzca aprendizaje; es evidente que siempre la capacidad colectiva supera a la de un miembro individual de ese mismo colectivo (Mann \& Helbing, 2017). Actualmente, el profesorado suministra el conocimiento y cuando se pide al alumnado que cree conocimiento es para evaluarlo, no para que se produzca aprendizaje.

La gestión del conocimiento creado por todos los miembros de una organización a través de un proceso de aprendizaje exige la cooperación de los individuos. La historia y evolución de la humanidad se ha basado en la cooperación de los individuos y conocimiento. Uno de esos estudios se basa en la sociología y las ciencias de la computación, es la denomina inteligencia colectiva.

Esta idea se ha aplicado diversas áreas en el contexto educativo, como en medicina para mejorar los diagnósticos (Kämmer, Hautz, Herzog, Kunina-Habenicht, \& Kurvers, 2017), para la toma de decisiones (De Vincenzo, Giannoccaro, Carbone, \& Grigolini, 2017), por tanto, la inteligencia colectiva ya ha sido trabajada en la escuela.

Otro impulsor importante de la transformación de la sociedad industrial a la del conocimiento son las tecnologías de la información y las comunicaciones (TIC). Las TIC están diseñadas para trabajar y gestionar información y conocimiento (García-Peñalvo, 2016). Por tanto, son un soporte idóneo para compartir y organizar el conocimiento de las distintas personas de una organización (García-Peñalvo, 2011).

Otro enfoque de la cooperación para generar conocimiento es el denominado inteligencia colectiva, que se centra en el estudio de la inteligencia grupal obtenida a partir de la inteligencia individual del colectivo. Comparte los mismos principios que la gestión del conocimiento de Nonaka y Takeuchi (1995) pero se encarga más de estudiar las relaciones del grupo y el comportamiento del conocimiento generado por el mismo, bajo la óptica de que la capacidad del colectivo siempre supera a la del individuo (Mann \& Helbing, 2017).

Los principios de la inteligencia colectiva se pueden aplicar a cualquier grupo de alumnos matriculados en una asignatura, ya que comparten unos mismos objetivos (aprobar la asignatura), tienen distintos niveles de conocimiento sobre la materia de la asignatura, se tiene acceso canales para poder cooperar y se pueden compartir diversas tareas y procesos.

Este trabajo se corresponde con una investigación para crear inteligencia colectiva entre el alumnado de una misma asignatura a partir de la compartición de conocimiento y procesos propios del aprendizaje que se produce en una asignatura universitaria.

Se pretende identificar el tipo de conocimiento que se puede compartir, las ventajas y los problemas que plantea la consideración de un proceso de inteligencia colectiva en el aula.

\section{CONTEXTO.}

La investigación se aplica en la asignatura de informática y programación, correspondiente al primer curso del grado de Ingeniería de la Energía de la Universidad Politécnica de Madrid. La asignatura tiene una cargad de 6 créditos, de los cuales 2 se dedican a impartir un laboratorio de programación en aulas de informática. Los grupos son de 30 alumnos.

La investigación se aplicó en un grupo de laboratorio de programación en Matlab para la resolución de Métodos Numéricos.

\section{DESCRIPCIÓN.}

Se creó una red social privada para el alumnado y profesorado de cada uno de los grupos donde se realizó la investigación. La red social elegida fue Google +, el motivo de dicha elección se debe a que es una red social popular y por disponer de apartados donde ubicar los distintos recursos generados por las personas participantes en la red social.

El laboratorio se organiza a través de nueve sesiones, por tanto se creó una organización que asociase los recursos creados en cada sesión al número de sesión. En la red social google + la creación de apartados se hace a través de la opción filtros, cada filtro equivale al nombre de la sesión (ver Figura 1-a). Si se pulsa en el nombre de un filtro, por ejemplo, sesión 3 , se muestran únicamente los recursos que se han creado en dicha sesión, la Figura 1 muestra dicha situación.

Así mismo se pretende clasificar el tipo de recurso que aporta cada miembro de la red. La clasificación definida antes de comenzar se agrupó en tres categorías “Acción, Temática y Fuente” y cada categoría, a su vez, tiene un conjunto de etiquetas. El listado completo se puede ver en la Figura 1-b.

Cada vez que una persona sube un recursos a la vez se muestra la siguiente información: usuario (información que por defecto genera la red), filtro (sesión) la red obliga a elegir uno antes de hacer público el recursos y las etiquetas (la red da opción de ponerlas o no). Así pues, cuando se sube un recurso se puede observar la sesión (Figura 1-a) y las etiquetas que ha asignado el usuario (Figura 1-c).

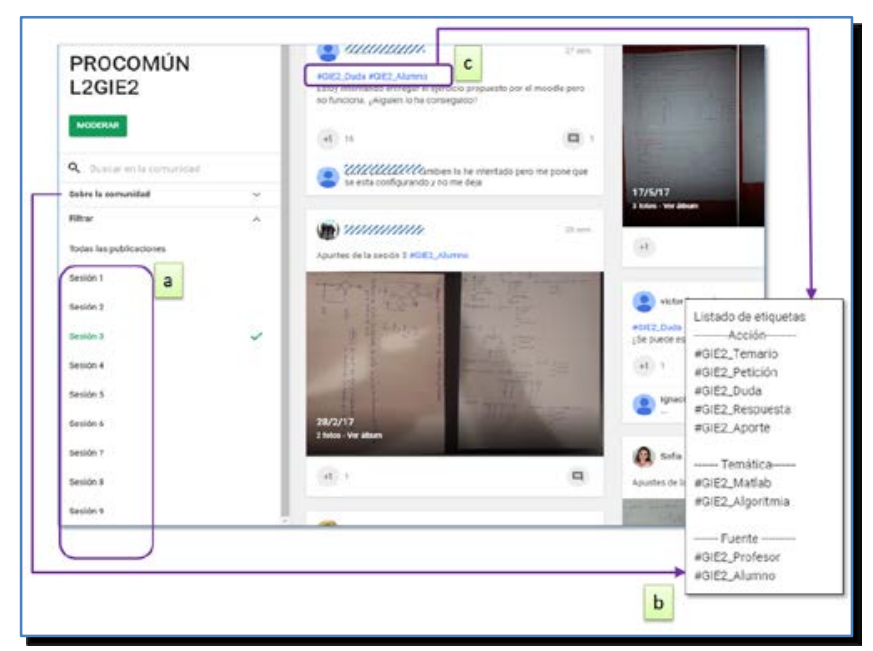

Figura 1. Organización del espacio

El proceso para cada sesión es el siguiente:

- De forma previa a la sesión el profesorado sube la planificación de la sesión. A este recuso se le denomina temario (Figura 2).

- Durante la clase presencial el alumnado sube a la red y comparte en tiempo real dudas, aclaraciones, ejemplos, resolución de problemas y cualquier otra información que considere fruto de su aprendizaje; 
por ejemplo, explica una duda que le ha sido resuelta o una dificultad que el mismo resolvió (Figura 3).

- $\quad$ Posteriormente a la clase la red se continua utilizando para que los alumnos continúen con las dudas, compartan sus apuntes o cualquier otra información fruto del aprendizaje que están realizando fuera del aula (Figura 4).

- El profesorado supervisa los recursos y los asocia al temario inicial de la sesión (Figura 2).

Angel Fidalgo Propietario , PROCOMÚN L2GIE2 $\vee \quad 8$ mar. 2017

\#GIE2_Temario \#GIE2_Profesor

SESIÓN 4. GRÁFICAS MATLAB.

0- Lo que se debe saber.

Entender cualquier programa escrito en Matlab con las funciones básicas.

1. Truco.

Función num2str

https://drive.google.com/file/d/0Bw4g7rdUea1TcTZXVHJwRjNjdDg/view? usp=sharing

2- Gráficas en Matlab

Entendiendo el concepto.

https://drive.google.com/file/d/0Bw4g7rdUea1TSFZUYmxCVmpUVFE/view?

usp=sharing

Vamos con un ejercicio.

https://drive.google.com/file/d/OBw4g7rdUea1TdDVVVnBmTjJuYzg/view?

usp=sharing

3. Gráfica de Fermat

https://drive.google.com/file/d/OBw4g7rdUea1TTG1xcWFGNDdnN1U/view?

usp=sharing

4- Trabajando con matrices.

Suma de matrices.

http://138.4.83.162/organiza/sicweb1/ParticularVer.asp?P=26 2910\&HT=1 elementos

https://drive.google.com/file/d/0Bw4g7rdUea1TcFBmaThwd3Uyd0k/view? usp=sharing

5- Problema propuesto.

$$
+1 \quad 3
$$

Figura 2. Recurso temario subido por el profesor. Los apartados de texto negro se corresponden con el temario subido de forma previa a la sesión. Los enlaces de cada apartado se corresponden con los recursos surgidos durante la sesión presencial, dichos recursos se asocian al temario inicial.

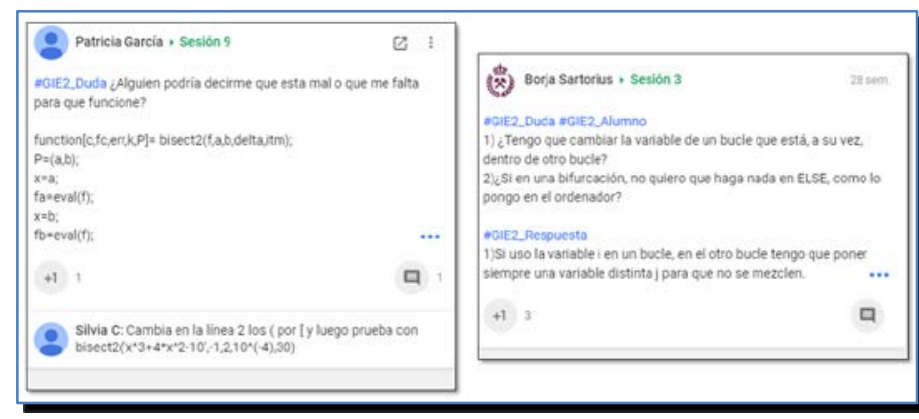

Figura 3. Ejemplos de dudas. Duda planteada por una alumna y resuelta por otra (izquierda) y duda resuelta por la misma persona que la planteó (derecha).

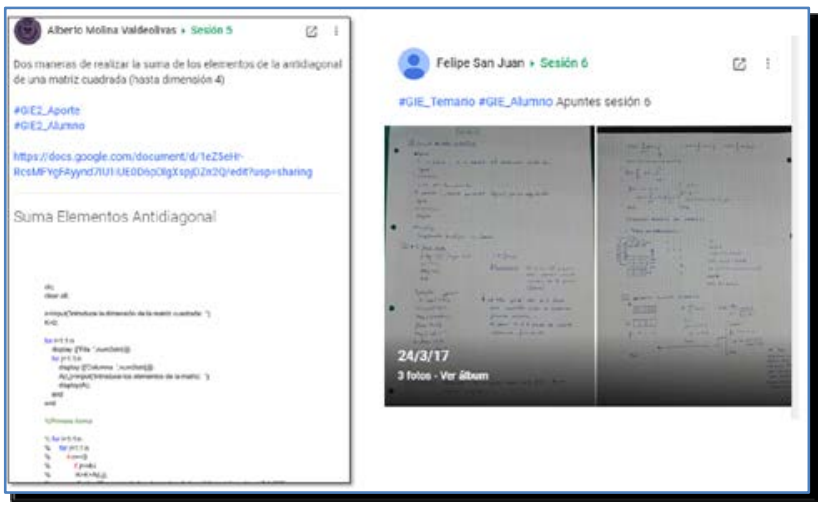

Figura 4. Ejemplos de aportes que realizan los alumnos con posterioridad a las clases presenciales. Resolución de un problema (izquierda) y apuntes (derecha).

Este planteamiento se realizó durante todas las sesiones que duró el laboratorio y la red se ha dejado abierta de forma permanente.

\section{RESULTADOS}

Se han obtenido datos cualitativos a través de una encuesta realizada a los participantes en la red social equivalente a uno de los grupos. En dicha encuesta han participado 31 alumnos que equivale al $100 \%$ de los alumnos participantes en la experiencia.

\section{Datos cualitativos.}

Se ha realizado una encuesta con 9 preguntas. La encuesta está estructurada en tres dimensiones: los recursos, la red y los contenidos. Cada dimensión tiene asociada tres preguntas. Las dos primeras dimensiones serían aplicables en general a cualquier asignatura. La tercera dimensión tiene por objetivo mejorar los contenidos del laboratorio y por tanto solamente sería aplicables en asignaturas donde hubiese laboratorios de programación en Matlab.

Las tres primeras preguntas (Q1-Q3) miden la utilización de los recursos aportados en la red social a través de una escala Lykert de 4 puntos (1 nada de acuerdo, 2 algo de acuerdo, 3 bastante de acuerdo y 4 muy de acuerdo). Su objetivo es comprobar el uso, utilidad y calidad. Las preguntas de esta dimensión su resultado están en las tablas 1, 2 y 3 .

Tabla 1. Uso de los recursos

Q1. He leído algún contenido correspondiente a las diferentes sesiones de clase.

\begin{tabular}{cccccc}
\hline $\begin{array}{c}\text { Apuntes } \\
\text { aportados } \\
\text { compañeros }\end{array}$ & por & $13 \%$ & $28 \%$ & $41 \%$ & $19 \%$ \\
\hline $\begin{array}{c}\text { Dudas } \\
\text { Respuestas }\end{array}$ & y & $6 \%$ & $31 \%$ & $31 \%$ & $31 \%$ \\
\hline $\begin{array}{c}\text { Temario } \\
\text { profesor }\end{array}$ & del & $0 \%$ & $3 \%$ & $47 \%$ & $50 \%$ \\
\hline $\begin{array}{c}\text { Programas } \\
\text { resueltos } \\
\text { compañeros }\end{array}$ & por & $9 \%$ & $13 \%$ & $41 \%$ & $38 \%$ \\
\hline
\end{tabular}


Tabla 2. Utilidad de los recursos

Q2. Indica el grado de utilidad de los siguientes contenidos

\begin{tabular}{cccccc}
\hline $\begin{array}{c}\text { Apuntes } \\
\text { aportados } \\
\text { compañeros }\end{array}$ & por & $16 \%$ & $50 \%$ & $28 \%$ & $6 \%$ \\
\hline $\begin{array}{c}\text { Dudas } \\
\text { Respuestas }\end{array}$ & y & $0 \%$ & $19 \%$ & $56 \%$ & $25 \%$ \\
\hline $\begin{array}{c}\text { Temario del } \\
\text { profesor }\end{array}$ & $0 \%$ & $13 \%$ & $41 \%$ & $47 \%$ \\
\hline $\begin{array}{c}\text { Programas } \\
\text { resueltos por } \\
\text { compañeros }\end{array}$ & $0 \%$ & $19 \%$ & $38 \%$ & $44 \%$ \\
\hline
\end{tabular}

Tabla 3. Preferencias revisión

Q3. Preferiría que los contenidos de la red social subidos de forma individual por un/a compañer@s fuese subido revisado previamente por un grupo de personas.

\begin{tabular}{|c|c|c|c|c|}
\hline Apuntes & $6 \%$ & $31 \%$ & $34 \%$ & $28 \%$ \\
\hline $\begin{array}{cc}\text { Dudas } & \mathbf{y} \\
\text { Respuestas } & \end{array}$ & $16 \%$ & $25 \%$ & $38 \%$ & $22 \%$ \\
\hline \begin{tabular}{l}
\multicolumn{1}{c}{ Programas } \\
resueltos \\
(verificando su \\
funcionamiento \\
por el profesor)
\end{tabular} & $9 \%$ & $13 \%$ & $31 \%$ & $47 \%$ \\
\hline
\end{tabular}

Las preguntas Q4-Q6 son preguntas abiertas que persiguen comprobar la eficacia del método así como su mejora, para ello se hacen tres preguntas: Q4-Indica lo qué es lo que más te ha gustado de la red social, Q5- Indica lo que menos te ha gustado de la red social y Q6- Indica qué mejorarías de la red social. Se ha realizado un análisis de las respuestas en abierto y posteriormente agrupadas en diversas categorías (ver Figura $5)$.

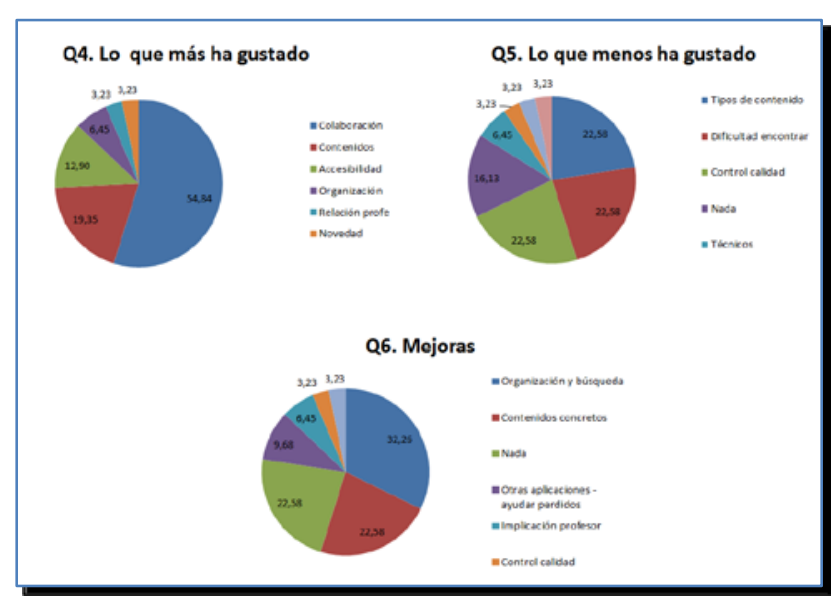

Figura 5. Resultados preguntas Q4 a Q5

Finalmente, las preguntas Q7, Q8 y Q9 se refieren a temas concretos del laboratorio, por lo que sus respuestas no se incluyen en este trabajo ya que únicamente aportan datos para mejorar la propia temática, no el método.

\section{CONCLUSIONES}

Los resultados de la pregunta Q1 arrojan que la mayor parte del alumnado ha accedido a los contenidos que se crean en la red. El 60\% indican que están muy o bastante de acuerdo con el acceso de los apuntes que ha generado el alumnado; el $62 \%$ las dudas y respuestas aportadas también por los participantes, el $79 \%$ los ejercicios resueltos por compañeros y de forma mayoritaria, el $97 \%$ los recursos que ha aportado el profesorado.

La segunda pregunta se basa en la utilidad de los contenidos; es decir, si les ha servido para el aprendizaje del laboratorio. En este caso el porcentaje (muy o bastante de acuerdo) es alto para las dudas y respuestas (81\%), para recursos aportados por el profesorado (88\%) y programas resueltos por el alumnado (81\%). Sin embargo es muy bajo para los apuntes aportados por los compañeros (34\%).

La tercera pregunta se pregunta por un posible control de calidad a los contenidos que se suben a la red social. El $62 \%$ opina que los apuntes deben estar revisados, el 60\% las dudas y respuestas y el $78 \%$ los ejercicios aportados por el alumnado, pero en este caso la revisión sería por parte del profesorado.

La conclusión de este primer bloque de tres preguntas demuestran que:

- El alumnado genera conocimiento (avalado por la utilidad de los recursos aportados) y, además, de forma habitual lo revisa.

- El alumnado apoya la creación de un control de “calidad” basado en la supervisión bien por pares o bien el propio profesorado.

- Los apuntes generados por el alumno es considerado como la más baja utilidad de todos los recursos aportados.

El segundo bloque de preguntas abiertas demuestra que lo más valorado de esta experiencia por parte del alumnado es la cooperación entre ellos, el 54,84\% de las respuestas en abierto lo indican. Con estos datos se valida se valida el principal principio dela inteligencia colectiva que es la cooperación.

Se demuestra que son varias las causas que menos gustan al alumnado: los contenidos (la baja calidad o utilidad de algunos contenidos subidos), la dificultad de encontrar esos contenidos (problema asociado a la gestión de las redes sociales) y el que no hay control de calidad de lo que se aporta. Básicamente estos datos se agrupan en un técnico que es un problema general de todas las redes sociales y, por otro, que no haya ninguna acreditación de la validez de los contenidos aportados por el alumnado. Este dato se complementa con los resultados de la pregunta Q2.

Finalmente, destaca que el 22,58 del alumnado no cree que sean necesarias mejoras; el mismo porcentaje que indica la mejora de un contenido concreto (hay mucha dispersión en el contenido concreto a mejorar) y la mayoría el 32,26\% destaca la necesidad de una mejora técnica en el sistema de búsqueda de contenidos.

\section{AGRADECIMIENTOS.}


Los autores quieren agradecer al proyecto de Innovación Educativa con código IE1617.0602 de la Universidad Politécnica de Madrid. Al Gobierno de Aragón, el Fondo Social Europeo, y el Ministerio de Educación de la Región de Castilla y León por su apoyo, así como a los grupos de investigación (LITI, http://www.liti.es; GIDTIC, http://gidtic.com y GRIAL, http://grial.usal.es).

Este trabajo está parcialmente financiado por el Ministerio de Economía y Competitividad del Gobierno de España a través del proyecto DEFINES (Ref. TIN2016-80172-R).

\section{REFERENCIAS}

De Vincenzo, I., Giannoccaro, I., Carbone, G., \& Grigolini, P. (2017). Criticality triggers the emergence of collective intelligence in groups. Physical Review E, 96(2), $\quad 022309$. doi:10.1103/PhysRevE.96.022309

Fidalgo-Blanco, Á., Sein-Echaluce, M. L., \& García-Peñalvo, F. J. (2014). Knowledge Spirals in Higher Education Teaching Innovation. International Journal of Knowledge Management, 10(4), 16-37. doi:10.4018/ijkm.2014100102

Fidalgo-Blanco, Á., Sein-Echaluce, M. L., \& García-Peñalvo, F. J. (2015). Epistemological and ontological spirals: From individual experience in educational innovation to the organisational knowledge in the university sector. Program: Electronic library and information systems, 49(3), 266-288. doi:10.1108/PROG-062014-0033

García-Peñalvo, F. J. (2011). La Universidad de la próxima década: La Universidad Digital. In C. SuárezGuerrero \& F. J. García-Peñalvo (Eds.), Universidad y Desarrollo Social de la Web (pp. 181-197). Washington DC, USA: Editandum.

García-Peñalvo, F. J. (2016). La socialización como proceso clave en la gestión del conocimiento. Education in the Knowledge Society, 17(2), 7-14. doi:http://dx.doi.org/10.14201/eks2016172714

Kämmer, J. E., Hautz, W. E., Herzog, S. M., KuninaHabenicht, O., \& Kurvers, R. H. J. M. (2017). The Potential of Collective Intelligence in Emergency Medicine: Pooling Medical Students' Independent Decisions Improves Diagnostic Performance. Medical Decision Making, 37(6), 715-724. doi:10.1177/0272989X17696998

Mann, R. P., \& Helbing, D. (2017). Optimal incentives for collective intelligence. Proceedings of the National Academy of Sciences of the United States of America, 114(20), 5077-5082. doi:10.1073/pnas.1618722114

Nonaka, I., \& Takeuchi, H. (1995). The knowledge creating company. New York, NY: Oxford University Press.

Pör, G. (2008). Collective Intelligence and Collective Leadership: Twin Paths to Beyond Chaos. Sprouts: Working Papers on Information Systems, 8(2).

Ramírez-Montoya, M. S., \& García-Peñalvo, F. J. (2018). Cocreation and open innovation: Systematic literature review. Comunicar, 26(54).

Sein-Echaluce, M. L., Fidalgo-Blanco, Á., \& García-Peñalvo, F. J. (2016). Students' Knowledge Sharing to improve Learning in Engineering Academic Courses. International Journal of Engineering Education (IJEE), 32(2B), 1024-1035.

Siemens, G. (2005). Connectivism: A learning theory for the digital age. International Journal of Instructional Technology and Distance Learning, 2(1), 3-10. 